\title{
BMJ Open Multimorbidity and patterns of chronic conditions in a primary care population in Switzerland: a cross-sectional study
}

Anouk Déruaz-Luyet, ${ }^{1}$ A Alexandra N'Goran, ${ }^{1}$ Nicolas Senn, ${ }^{1}$ Patrick Bodenmann, ${ }^{2}$ Jérôme Pasquier, ${ }^{3}$ Daniel Widmer, ${ }^{1}$ Ryan Tandjung, ${ }^{4}$ Thomas Rosemann, ${ }^{4}$ Peter Frey, ${ }^{5}$ Sven Streit, ${ }^{5}$ Andreas Zeller, ${ }^{6}$ Dagmar M Haller, ${ }^{7}$ Sophie Excoffier, ${ }^{7}$ Bernard Burnand, ${ }^{3}$ Lilli Herzig ${ }^{1}$

To cite: Déruaz-Luyet $A$, N'Goran AA, Senn N, et al. Multimorbidity and patterns of chronic conditions in a primary care population in Switzerland: a cross-sectional study. BMJ Open 2017;7:e013664. doi:10.1136/ bmjopen-2016-013664

- Prepublication history and additional material for this paper are available online. To view these files please visit the journal online (http://dx.doi. org/10.1136/bmjopen-2016013664).

Received 29 July 2016

Revised 28 March 2017

Accepted 30 March 2017

\section{CrossMark}

${ }^{1}$ Institute of Family Medicine, University of Lausanne, Lausanne, Switzerland ${ }^{2}$ Department of Ambulatory Care and Community Medicine, Lausanne University Hospital,

Lausanne, Switzerland

${ }^{3}$ Institute of Social and Preventive Medicine, Lausanne University Hospital, Lausanne,

Switzerland

${ }^{4}$ Institute of Primary Care, University of Zurich, Zurich, Switzerland

${ }^{5}$ Institute of Primary Health Care (BIHAM), University of Bern, Bern, Switzerland

${ }^{6}$ Centre for Primary Health Care, University of Basel, Basel, Switzerland

${ }^{7}$ Primary Care Unit, Faculty of Medicine, University of Geneva, Geneva, Switzerland

Correspondence to

Dr Lilli Herzig; lilli.herzig@hin.ch

\section{ABSTRACT}

Objective To characterise in details a random sample of multimorbid patients in Switzerland and to evaluate the clustering of chronic conditions in that sample.

Methods 100 general practitioners (GPs) each enrolled 10 randomly selected multimorbid patients aged $\geq 18$ years old and suffering from at least three chronic conditions. The prevalence of 75 separate chronic conditions from the International Classification of Primary Care-2 (ICPC-2) was evaluated in these patients. Clusters of chronic conditions were studied in parallel.

Results The final database included 888 patients. Mean (SD) patient age was 73.0 (12.0) years old. They suffered from 5.5 (2.2) chronic conditions and were prescribed 7.7 (3.5) drugs; $25.7 \%$ suffered from depression. Psychological conditions were more prevalent among younger individuals ( $\leq 66$ years old). Cluster analysis of chronic conditions with a prevalence $\geq 5 \%$ in the sample revealed four main groups of conditions: (1) cardiovascular risk factors and conditions, (2) general age-related and metabolic conditions, (3) tobacco and alcohol dependencies, and (4) pain, musculoskeletal and psychological conditions.

Conclusion Given the emerging epidemic of multimorbidity in industrialised countries, accurately depicting the multiple expressions of multimorbidity in family practices' patients is a high priority. Indeed, even in a setting where patients have direct access to medical specialists, GPs nevertheless retain a key role as coordinators and often as the sole medical reference for multimorbid patients.

\section{INTRODUCTION}

Multimorbidity occurs when one person suffers from two or more chronic medical conditions without any single condition being considered the main one. ${ }^{1}$ Caring for multimorbid patients is an important part of general practice. A large, population-based study conducted in the UK, which included children, estimated that $23.2 \%$ of the population visiting a general practitioner (GP) had at least two chronic conditions. ${ }^{2}$ Estimates of the prevalence of multimorbidity in

\section{Strengths and limitations of this study}

- First Swiss study involving multimorbid patients in primary care, aged 18 years old and over, and enrolled across five large regions of the country.

- High-quality database on a random sample of 888 patients seen in 100 general practitioners' (GPS) practices, with information collected from both patients and GPs.

- Use of a list of 75 chronic conditions from the International Classification of Primary Care-2 classification to represent the variety and complexity of the chronic conditions seen by GPs.

- Evaluation of the prevalence of the 75 conditions across the entire patient sample and a subsample of younger patients ( $\leq 66$ years old), although some subsample estimates were based on very few observations.

- Identification of four clusters of chronic conditions, underlining the multiplicity of multimorbidity profiles. The sample size and the high number of conditions considered did not permit cluster analyses stratified by gender or age group.

Switzerland's primary care populations have varied between $13.0 \%$ and $76.6 \%$, depending on the chronic conditions considered, the data used and the population covered. ${ }^{3-6}$ The prevalence and outcomes of multimorbidity are influenced by different factors, including age and socioeconomic status. ${ }^{278}$

The effects of multiple chronic conditions on an individual are complex. Specific combinations of conditions may have greater effects on functional status, quality of life and mortality than others. ${ }^{9}{ }^{10}$ Recent studies have highlighted that some chronic conditions tend to co-occur more frequently than others $^{11}$ due to either a high prevalence of these individual conditions within the population or common pathophysiological mechanisms. ${ }^{12}$ Treatment management for multimorbid patients can be very complex 
due to numerous different medications, potential drug interactions and numerous healthcare partners. In addition, treatment guidelines are typically developed for one condition and do not apply to multimorbidity. ${ }^{2913}$ Enhanced understanding of the patterns of chronic-disease clustering would help to improve the management of multimorbid patients and to define better-adapted treatment guidelines. ${ }^{12} 14$

We conducted a study to characterise patients with multimorbidity in primary care in Switzerland and to evaluate the clustering of chronic conditions in this sample of patients. To the best of our knowledge, this was the first study to describe a random sample of multimorbid patients in primary care on a national scale. Also, clusters of chronic conditions have not previously been examined in detail in the Swiss primary care population. To underline the complexity of multimorbidity in primary care, we considered 75 chronic conditions and did not restrict our study to older patients. We included adult patients with a minimum of three chronic conditions in order to target a population whose management would be more challenging to GPs.

\section{METHODS}

\section{Study design and participants}

The detailed study protocol has been published elsewhere. ${ }^{15}$ It was approved by the Human Research Ethics Committee of the Canton Vaud (Protocol 315/14). Briefly, we conducted a cross-sectional study involving a convenience sample of 100 GPs spread across five large regions of Switzerland. We aimed to enrol a random sample of 1000 adult patients (10 per GP) suffering from three or more chronic conditions from a list of 75 items/ codes identified in the International Classification of Primary Care-2 (ICPC-2) classification. ${ }^{16}$ Data collection began with the GPs completing a paper-questionnaire on demographic and private practice-related data (GP-related variables). GPs asked eligible patients to participate, and if they gave written informed consent, a research assistant conducted a post-consultation telephone interview (patient-related variables assessed through the patient survey). If an eligible patient refused to participate, then the GP documented his/her date of birth, sex and reason for refusing. The GP completed a paper questionnaire for each patient (patient-related variables assessed through the GP survey). All the variables have been described in the protocol. ${ }^{15}$ All patient data were coded during the data collection process.

\section{Data management}

Two researchers independently checked the database and reconciled their versions. Using a random sample of 44 questionnaires (about 5\% of the final database), the error rate in the final database of patient-related variables assessed through the GP survey was evaluated at $0.5 \%$. Finally, the error rate and the difference between the research assistants during the telephone interviews (patient-related survey) were evaluated by performing simultaneous data entry during 44 interviews (about 5\% of the sample). Their difference rate was measured at 2.7\%. Further analysis revealed that most of the discrepancy between research assistants arose from differences in coding responses to the Treatment Burden Questionnaire, one specific part of the patient survey ${ }^{17}$ Finally, during a subsample of 50 telephone interviews, the reliability of data was assessed, with each research assistant asked to complete a paper version of the patient survey in parallel to their direct data entry. The difference rate was $0.45 \%$.

\section{Statistical analyses}

All analyses were conducted using Stata software, V.14 (StataCorp), and R software, V.3.2.5 (Foundation for Statistical Computing). Associations were considered significant for $\mathrm{p}$ values $<0.05$.

\section{Description of the samples of GPs and patients}

Descriptive statistics of the samples of GPs and patients included in the study were calculated. The prevalence estimates per 100 people were computed for each of the 75 chronic conditions. The $95 \%$ CIs for these prevalence estimates were computed assuming a binomial distribution. For analytical relevance, some mutually exclusive conditions were considered together: both types of ischaemic heart disease (IHD) (K74 and K76) in the IHD category, both types of diabetes (D) (T89 and T90) in the $\mathrm{D}$ category and the three items relating to elevated blood pressure (BP) or hypertension (K85, K86 and K87) in the BP category. Prevalence estimates were then computed for the subgroup of younger patients taking the complete sample's first quartile of age as the upper age limit. The frequency of triads of conditions was evaluated to assess whether some conditions would co-occur more frequently than might be expected, based on each condition's individual prevalence in the sample. For this analysis, we considered the probability of a patient suffering from at least the triad of conditions considered in combination (see online supplementary table 1 for detailed method).

\section{Cluster of chronic conditions}

A cluster analysis was conducted to identify clinically relevant clusters of chronic conditions, based on their proximity among respondents. Data on chronic conditions were considered as binary, with a value of 1 when the condition was present and 0 when it was absent. As there was no a priori number of clusters, a hierarchical clustering approach was used, in which all the individual chronic conditions were first considered as clusters themselves and were then merged gradually with their most closely related clusters. The distances between conditions were measured using Yule's $Q$ distance ((1-Yule's $\mathrm{Q}$ correlation coefficient)/2), and the average linkage method was used to determine the distances between clusters. Results were assessed using a dendrogram. Chronic conditions with a prevalence $<5 \%$ were not considered in 
Table 1 Characteristics of the sample of GPs, $n=100$.

\begin{tabular}{|c|c|}
\hline Age (years) & Range: 31-76 \\
\hline Mean (SD) & $52.9(9.3)$ \\
\hline Male sex (n) & 72 \\
\hline \multicolumn{2}{|l|}{ Medical school (n) } \\
\hline In Switzerland & 97 \\
\hline In Germany & 2 \\
\hline In Mexico & 1 \\
\hline \multicolumn{2}{|l|}{ Board certification (n) } \\
\hline $\begin{array}{l}\text { Board certified in internal and family } \\
\text { medicine }\end{array}$ & 96 \\
\hline Not board certified, practicing physician & 4 \\
\hline GP with a second board certification (n) & 13 \\
\hline Other board certifications ( $\mathrm{n})$ & $15^{*}$ \\
\hline Angiology* & 2 \\
\hline Emergency hospital medicine* & 3 \\
\hline Endocrinology* & 1 \\
\hline Geriatrics* & 3 \\
\hline Nephrology* & 1 \\
\hline Occupational medicine ${ }^{\star}$ & 1 \\
\hline Palliative care* & 1 \\
\hline Sports medicine* & 3 \\
\hline $\begin{array}{l}\text { GP with other medical subspecialisation } \\
\text { (n) }\end{array}$ & 11 \\
\hline
\end{tabular}

$\begin{array}{lc}\text { Manual medicine } & 6 \\ \begin{array}{l}\text { Psychosomatic and psychosocial } \\ \text { medicine }\end{array} & 5 \\ \text { Type of practice }(\mathrm{n}) & \\ \text { Individual practice } & 29 \\ \text { Group practice } & 70 \\ \text { Practice within a retirement home } & 1 \\ \text { Member of a healthcare network }(n) & 68\end{array}$

Number of consultations/week (n)

\begin{tabular}{lc}
$<30$ & 2 \\
$>30-70$ & 25 \\
$>70-110$ & 47 \\
$>110-170$ & 22 \\
$>170$ & 4 \\
$\begin{array}{l}\text { Other activities (besides private practice) } \\
\text { (n) }\end{array}$ & $82 \dagger$ \\
$\begin{array}{l}\text { As referring physician in a company } \\
\text { As referring physician in a specific } \\
\text { environment (school, retirement home, } \\
\text { prison, community health centre) }\end{array}$ & 13 \\
$\begin{array}{l}\text { Involved in training/teaching medical } \\
\text { students or in research }\end{array}$ & 48 \\
$\begin{array}{l}\text { Other } \\
\text { Number of years since medical school } \\
\text { diploma }\end{array}$ & Range: 8-50 \\
\hline
\end{tabular}

Continued

\begin{tabular}{ll}
\hline Table 1 Continued & \\
\hline Mean (SD) & $26.9(9.4)$ \\
Practice location $\ddagger$ & \\
Urban & 36 \\
Suburban & 44 \\
Rural & 20
\end{tabular}

*Two GPs had two other board certifications.

†Some GPs had more than one other activity.

$\ddagger$ Data on location for each postal code were obtained from Swiss

Federal Statistical Office.

GP, general practitioner.

this analysis in order to avoid biases and false associations. For the same reasons, our patient sample size and the high number of conditions considered did not allow for a reliable cluster analysis stratified by gender, age group or socioeconomic status.

\section{RESULTS}

\section{Sample of GPs}

A convenience sample of 100 GPs was assembled; their characteristics are detailed in table 1. The sample was fairly representative of the population of all GPs practising in Switzerland ${ }^{1819}$ in terms of sex $(72 \%$ male vs $78 \% ; \mathrm{p}=0.26)$. It comprised similar proportions of GPs

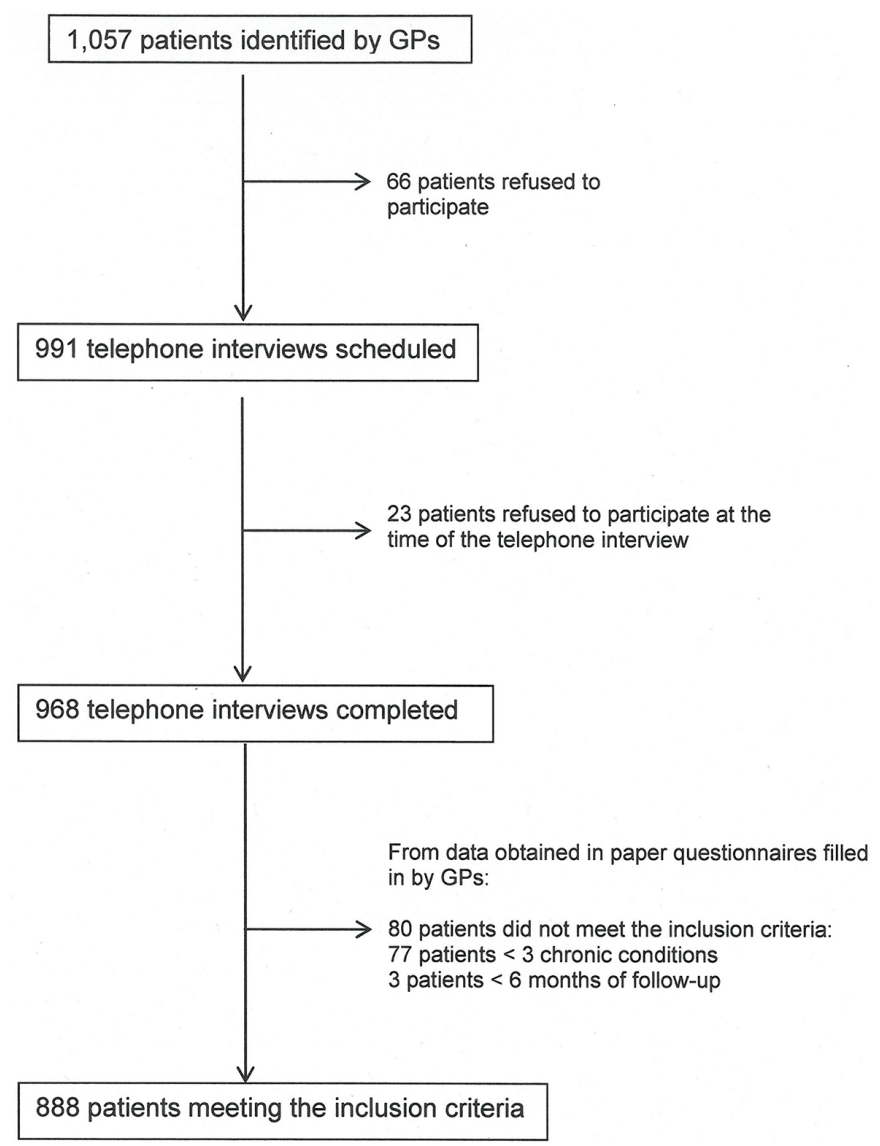

Figure 1 Patient inclusion flowchart. GP, general practitioner. 
practising in urban $(36 \%)$, suburban $(44 \%)$ and rural $(20 \%)$ areas $(p=0.28)$. However, the average age of the present study's GPs was lower (52.9 vs 55.0 years old; $\mathrm{p}<0.05$ ), and they were more likely to work in a group practice $(70 \%$ vs $52.3 \% ; \mathrm{p}<0.01)$. This mirrors the tendency for younger GPs in Switzerland to work in group practices. ${ }^{20}$ GPs had been practising medicine for an average of 26.9 years (SD 9.4).

\section{Sample of patients}

A study flowchart is represented in figure 1. Of the 1057 patients approached, 6.2\% (66 patients) refused to participate. Reasons for refusal were that patients felt too sick $(43.9 \%)$, were not interested $(31.8 \%)$, had no time $(6.1 \%)$ or had other reasons $(56.1 \%)$. Among those other reasons, some patients mentioned that participating was too frightening/stressful (13.5\%), that they disliked telephone interviews $(16.2 \%)$ or that they had a hearing impairment or deafness preventing a telephone interview $(8.1 \%)$. Patients who refused to participate were not significantly different from those who did, in terms of either age $(\mathrm{p}=0.16)$ or $\operatorname{sex}(\mathrm{p}=0.25)$.

Of the 991 patients who initially agreed to take part in the study, $23(2.3 \%)$ refused participation at the time of the telephone interview. These patients were not significantly different from the final sample of patients in terms of age $(\mathrm{p}=0.46)$, sex $(\mathrm{p}=0.66)$, number of chronic conditions $(\mathrm{p}=0.11)$ or number of years of follow-up by the GP $(p=0.28)$. Eighty patients were removed from the sample after the telephone interview as they did not meet the inclusion criteria.

The final sample consisted of the 888 patients whose characteristics are reported in table 2. On average, patients with multimorbidity were 73 years old, married (49.2\% of the sample), lived in a household of two adults $(51.0 \%)$, without children $(95.5 \%)$ and were not receiving home care $(89.4 \%)$. They suffered from five to six chronic conditions (mean 5.5, SD 2.2), were prescribed seven to eight medications (mean 7.7, SD 3.5) and had been followed by their GP for about 11 years (median 9 years, IQR 13). One-fourth of the patients interviewed reported that their GP was the only medical doctor involved in their treatment.

\section{Prevalence of chronic conditions}

Of the 75 chronic conditions considered (see online supplementary table 2), 74 were observed at least once (all but condition N74-Malignant neoplasm, nervous system). Twenty-four conditions had a prevalence $\geq 5 \%$ in the sample (table $3 \mathrm{~A}$ ). We also evaluated the prevalence of all the chronic conditions in the subgroup of patients $\leq 66$ years old $(\mathrm{n}=234)$ (table $3 \mathrm{~B}$ ). Of the 24 conditions with a prevalence $\geq 5 \%$ in the overall sample, 22 also had a prevalence $\geq 5 \%$ in this subgroup of younger patients. Only atrial fibrillation and malignant neoplasm of the prostate were not as prevalent among younger individuals. Four conditions-migraine, chronic bronchitis, personality disorders and phobia or compulsive disorders-had a prevalence $\geq 5 \%$ in the subgroup of younger individuals but did not reach this level when all ages were considered.

\section{Cluster analysis}

Results from the cluster analysis of the whole sample for the 24 chronic conditions with a prevalence $\geq 5 \%$ are reported in Figure 2. Four main clusters of chronic conditions were defined in these analyses: (1) cardiovascular disease risk factors and cardiovascular conditions, (2) general age-related conditions and conditions relating to metabolic syndromes, (3) tobacco-related and alcohol-related dependencies and chronic obstructive pulmonary disease and (4) general pain, musculoskeletal and psychological conditions.

\section{DISCUSSION}

We reported the first results of a nationwide cross-sectional study on multimorbidity in primary care in Switzerland. The overall participation rate was high, and the database obtained was of high quality. We enrolled 888 patients with 3-19 chronic conditions (mean 5.5), taking from 0 to 21 medications (mean 7.7). The mean follow-up by a GP was 11 years. A quarter of patients reported being treated only by their GP, with no involvement from other medical specialists. Overall, $89 \%$ of patients were not receiving home care. Of the 75 chronic conditions considered, 74 were observed at least once. This is a reflection of the real complexity of multimorbidity in primary care settings and hints at the challenge that managing such patients can be. A cluster analysis based on the conditions in the sample with a prevalence of at least $5 \%$ revealed four main clusters: cardiovascular disease risk factors and conditions, age-related and metabolic conditions, tobacco-related and alcohol-related conditions and pain, musculoskeletal and psychological conditions.

The definition of multimorbidity, as well as the number of chronic conditions considered, varies greatly between studies and prevents a direct comparison with our results. ${ }^{21}$ Notably, the number of chronic conditions considered is generally from 5 to 40 . The management of patients with multimorbidity is challenging because of the interactions between the different chronic conditions within one person. In this context, GPs must set treatment priorities. Recently, disease interactions between highly prevalent chronic conditions have been described, leading to some appropriately adapted clinical recommendations. ${ }^{14}$ Although it is probably important for researchers and policy makers to concentrate on evaluating the problem of multimorbidity based on the most prevalent conditions, GPs, in contrast, have to deal with a wide variety of prevalent and less prevalent conditions. Their work is also often restricted by the absence of evidence for different associations between chronic conditions that are simply not described in the literature. In an attempt to reflect this high complexity, we used a list of 75 chronic conditions (based on the ICPC-2), 
Table 2 Sample of patients included in the study, $n=888$

\section{Demographic characteristics}

\begin{tabular}{|c|c|c|c|}
\hline \multicolumn{2}{|l|}{ Age (years) } & \multicolumn{2}{|c|}{ Number of chronic conditions diagnosed } \\
\hline Range & $28-98$ & Range & $3-19$ \\
\hline Mean (SD) & $72.95(12.0)$ & Mean (SD) & $5.5(2.2)$ \\
\hline \multicolumn{2}{|l|}{ Sex, n (\%) } & \multicolumn{2}{|c|}{ Number of medications prescribed } \\
\hline Male & $428(48.2)$ & Range & $0-21$ \\
\hline \multicolumn{2}{|l|}{ Marital status, n (\%) } & Mean (SD) & $7.7(3.5)$ \\
\hline Single & $85(9.6)$ & \multicolumn{2}{|c|}{ Number of substances prescribed } \\
\hline Married & $437(49.2)$ & Range & $0-22$ \\
\hline Separated/Divorced & $150(16.9)$ & Mean (SD) & $8.5(3.8)$ \\
\hline Widowed & $216(24.3)$ & \multicolumn{2}{|c|}{ Number of visits to the GP in the last 12 months* } \\
\hline \multicolumn{2}{|c|}{ Size of household-number of adults, $n(\%)$} & Range & $1-80$ \\
\hline 1 & $375(42.2)$ & Mean (SD) & $12.9(8.7)$ \\
\hline 2 & $453(51.0)$ & \multicolumn{2}{|c|}{ Number of visits to the GP in the last month } \\
\hline 3 & $48(5.4)$ & Range & $1-21$ \\
\hline 4 & $12(1.4)$ & Mean (SD) & $2.0(1.8)$ \\
\hline \multicolumn{2}{|c|}{ Size of household-number of children, ${ }^{*} n(\%)$} & \multicolumn{2}{|c|}{ Number of years of follow-up by the GP* } \\
\hline 0 & $848(95.5)$ & Range & $0.5-40$ \\
\hline 1 & $22(2.5)$ & Mean (SD) & $10.9(8.3)$ \\
\hline 2 & $14(1.6)$ & \multicolumn{2}{|c|}{ Receiving home care, $\mathrm{n}(\%)$} \\
\hline 3 & $3(0.3)$ & Yes & $94(10.6)$ \\
\hline 4 & $1(0.1)$ & & \\
\hline \multicolumn{2}{|l|}{ First language, $\mathrm{n}(\%)$} & \multicolumn{2}{|c|}{ Number of times a week } \\
\hline French & $282(31.8)$ & Range & $0.5-7.0$ \\
\hline German & $526(59.2)$ & Mean (SD) & $2.5(2.2)$ \\
\hline French and German & $8(0.9)$ & Receiving cle & \\
\hline Other & $72(8.1)$ & Yes, n (\%) & $236(26.6)$ \\
\hline \multicolumn{2}{|l|}{ Level of schooling, $n(\%)$} & \multicolumn{2}{|c|}{ Meal delivery services, $\mathrm{n}(\%)$} \\
\hline Primary school/no diploma & $130(14.6)$ & Yes & $53(6.0)$ \\
\hline Secondary school & $65(7.3)$ & \multicolumn{2}{|c|}{ Number of medical doctors involved, $n(\%)$} \\
\hline Practical vocational training & $225(25.3)$ & 1 & $237(26.7)$ \\
\hline $\begin{array}{l}\text { High school diploma/ } \\
\text { equivalent }\end{array}$ & $112(12.6)$ & 2 & $272(30.6)$ \\
\hline Professional school & $246(27.7)$ & 3 & $202(22.8)$ \\
\hline $\begin{array}{l}\text { Superior non-university } \\
\text { degree }\end{array}$ & $65(7.3)$ & 4 or more & 177 (19.9) \\
\hline University degree & $44(5.0)$ & Pillbox use, & \\
\hline \multicolumn{2}{|l|}{ Schooled in Switzerland, n (\%) } & Yes & $405(45.6)$ \\
\hline Yes & 753 (84.8) & & \\
\hline
\end{tabular}

*Some numbers do not add up to 888 because of missing data.

GP, general practitioner.

as determined by experts in primary care in Switzerland. ${ }^{16}$ By using this list, our sample of multimorbid patients was closer to that encountered by GPs in their daily practice than studies based on more limited lists.

As the prevalence of multimorbidity increases with age, most studies on this topic have included either

\section{General patient management}

Number of chronic conditions diagnosed

Number of medications prescribed

Number of substances prescribed

Number of visits to the GP in the last 12 months ${ }^{*}$

Number of visits to the GP in the last month

Number of years of follow-up by the GP*

Receiving home care, $\mathrm{n}(\%)$

Number of times a week

Receiving cleaning help, $\mathrm{n}(\%)$

Meal delivery services, $n(\%)$

$53(6.0)$

Number of medical doctors involved, $\mathrm{n}(\%)$

$3202(22.8)$

4 or more $\quad 177(19.9)$

Pillbox use, $\mathrm{n}(\%)$

Yes $405(45.6)$

older people (eg, 65 years old or older) ${ }^{22-26}$ or defined multimorbidity as the presence of at least two chronic conditions. ${ }^{21}$ The age of the multimorbid patients in our sample was not restricted, precisely because we wished to evaluate the age heterogeneity of this population and to assess the presence of multimorbidity in 
Table 3 Twenty-four conditions with a prevalence $\geq 5 \%$ in the sample of patients $(n=888)(A)$ and twenty-six conditions with a prevalence higher than $5 \%$ in the subgroup of patients $\leq 66$ years $(n=234)(B)$

\begin{tabular}{|c|c|c|c|}
\hline Condition & ICPC-2 code & $\begin{array}{l}\text { No of } \\
\text { observations }\end{array}$ & Prevalence $(\%)(95 \% \mathrm{Cl})$ \\
\hline \multicolumn{4}{|l|}{ (A) } \\
\hline $\mathrm{BP}$ & K85, K86, K87 & 657 & 74.0 (71.0 to 76.8$)$ \\
\hline $\begin{array}{l}\text { Cardiovascular disease risk factors including lipid } \\
\text { disorders }\end{array}$ & K22 & 359 & 40.4 (37.2 to 43.7$)$ \\
\hline Diabetes (D) & T89, T90 & 277 & $31.2(28.2$ to 34.4$)$ \\
\hline Obesity & T82 & 274 & $30.9(27.8$ to 34.0$)$ \\
\hline IHD & K74, K76 & 258 & 29.1 (26.1 to 32.2) \\
\hline Depressive disorders & P76 & 228 & 25.7 (22.8 to 28.7 ) \\
\hline Osteoarthrosis of the knee & L90 & 223 & 25.1 (22.3 to 28.1$)$ \\
\hline Pain (general or multiple site) & $\mathrm{A} 01$ & 198 & 22.3 (19.6 to 25.2$)$ \\
\hline Atrial fibrillation & K78 & 195 & $22.0(19.3$ to 24.8$)$ \\
\hline Atherosclerosis/peripheral vascular disease & K92 & 159 & 17.9 (15.4 to 20.6$)$ \\
\hline Osteoporosis & L95 & 154 & 17.3 (14.9 to 20.0$)$ \\
\hline Osteoarthrosis of the hip & L89 & 152 & 17.1 (14.7 to 19.8$)$ \\
\hline Chronic obstructive pulmonary disease & R95 & 119 & $13.4(11.2$ to 15.8$)$ \\
\hline Cerebrovascular disease & K91 & 106 & 11.9 (9.9 to 14.3$)$ \\
\hline Peripheral neuritis/neuropathy & N94 & 106 & 11.9 (9.9 to 14.3$)$ \\
\hline Hearing complaint, including deafness & $\mathrm{H} 02$ & 103 & 11.6 (9.6 to 13.9$)$ \\
\hline Gout & T92 & 97 & $10.9(8.9$ to 13.2$)$ \\
\hline Asthma & R96 & 83 & $9.3(7.5$ to 11.5$)$ \\
\hline Irritable bowel syndrome & D93 & 78 & $8.8(7.0$ to 10.8$)$ \\
\hline Tobacco abuse & $\mathrm{P} 17$ & 70 & $7.9(6.2$ to 9.9$)$ \\
\hline Rheumatoid arthritis/seropositive arthritis & L88 & 63 & 7.1 (5.5 to 9.0$)$ \\
\hline Incontinence (urine) & U04 & 60 & 6.8 (5.2 to 8.6$)$ \\
\hline Malignant neoplasm, prostate & Y77 & 49 & 5.5 (4.1 to 7.2$)$ \\
\hline Chronic alcohol abuse & P15 & 47 & 5.3 (3.9 to 7.0$)$ \\
\hline
\end{tabular}

(B)

\begin{tabular}{|c|c|c|c|}
\hline $\mathrm{BP}$ & K85, K86, K87 & 142 & 60.7 (54.1 to 67.0$)$ \\
\hline Obesity & T82 & 99 & 42.3 (35.9 to 48.9$)$ \\
\hline Depressive disorder & P76 & 93 & 39.7 (33.4 to 46.3$)$ \\
\hline $\begin{array}{l}\text { Cardiovascular disease risk factors including lipid } \\
\text { disorders }\end{array}$ & K22 & 92 & $39.3(33.0$ to 45.9$)$ \\
\hline Diabetes (D) & T89, T90 & 71 & $30.3(24.5$ to 36.7$)$ \\
\hline Pain (general or multiple site) & $\mathrm{A} 01$ & 68 & 29.1 (23.3 to 35.3$)$ \\
\hline Osteoarthrosis of knee & L90 & 46 & 19.7 (14.8 to 25.3$)$ \\
\hline Tobacco abuse & P17 & 41 & 17.5 (12.9 to 23.0$)$ \\
\hline Asthma & R96 & 33 & 14.1 (9.9 to 19.2$)$ \\
\hline IHD & $\mathrm{K} 74, \mathrm{~K} 76$ & 32 & 13.7 (9.5 to 18.8 ) \\
\hline Chronic obstructive pulmonary disease & R95 & 32 & 13.7 (9.5 to 18.8$)$ \\
\hline Chronic alcohol abuse & P15 & 28 & $12.0(8.1$ to 16.8$)$ \\
\hline Osteoporosis & L95 & 27 & $11.5(7.7$ to 16.3$)$ \\
\hline Irritable bowel syndrome & D93 & 26 & $11.1(7.4$ to 15.9$)$ \\
\hline Atherosclerosis/peripheral vascular disease & K92 & 24 & $10.3(6.7$ to 14.9$)$ \\
\hline Migraine & N89 & 23 & 9.8 (6.3 to 14.4$)$ \\
\hline
\end{tabular}




\begin{tabular}{|c|c|c|c|}
\hline Condition & ICPC-2 code & $\begin{array}{l}\text { No of } \\
\text { observations }\end{array}$ & Prevalence $(\%)(95 \% \mathrm{Cl})$ \\
\hline Cerebrovascular disease & K91 & 20 & 8.5 (5.3 to 12.9$)$ \\
\hline Gout & T92 & 19 & $8.1(5.0$ to 12.4$)$ \\
\hline Hearing complaint, including deafness & $\mathrm{H} 02$ & 18 & 7.7 (4.6 to 11.9$)$ \\
\hline Chronic bronchitis & $\mathrm{R} 79$ & 17 & $7.3(4.3$ to 11.4$)$ \\
\hline Personality disorder & P80 & 16 & 6.8 (4.0 to 10.9$)$ \\
\hline Osteoarthrosis of hip & L89 & 15 & 6.4 (3.6 to 10.4$)$ \\
\hline Incontinence urine & U04 & 14 & 6.0 (3.3 to 9.8$)$ \\
\hline Phobia/compulsive disorder & P79 & 12 & 5.1 (2.7 to 8.8$)$ \\
\hline
\end{tabular}

BP, elevated blood pressure or hypertension; ICPC-2, International Classification of Primary Care-2; IHD, ischaemic heart disease.

younger patients. We also chose to consider a minimum of three concurrent chronic conditions, as we were particularly interested in a population of patients that would prove more challenging to GPs. Despite the inclusion of younger patients ( $\geq 18$ years old), our sample consisted mostly of older individuals (mean age about 73 years old). Consequently, the most prevalent chronic conditions in our study included cardiovascular diseases and metabolic syndromes known to have a high occurrence in older adults. ${ }^{623-25} 2728$ Prevalence estimates obtained for high blood pressure/hypertension, diabetes, IHD and osteoporosis were similar to those obtained by van den Bussche $e t a l^{26}$ in a study of patients aged at least 65 years old and suffering from at least three chronic conditions identified from a list of 46 .

We observed a higher prevalence of depression in the present sample of multimorbid patients than those reported in some previous studies. ${ }^{24-26}$ We believe this could be an effect of considering younger multimorbid patients. The prevalence analysis on the subgroup of 234 patients aged $\leq 66$ years old showed a prevalence of depression of $39.7 \%$, compared with $25.7 \%$ when considering the entire sample $(\mathrm{p}<0.001$; table $3 \mathrm{~B})$, which supports this hypothesis. This subgroup of patients was also characterised by a higher prevalence of personality disorders and phobias or compulsive disorders. This observation is supported by previous studies on younger multimorbid patients, which reported depression/ anxiety $^{2129}$ or mental disorders ${ }^{21}{ }^{30}$ to be among the most prevalent chronic conditions. The size of this subgroup of patients did not allow for a complete analysis stratified by age as had been possible in studies including younger multimorbid primary care patients. ${ }^{21} 2728$

As we mentioned before, in primary care, multimorbidity can express itself in many ways, and defining the clustering of chronic conditions is a valuable aid to understanding the most common associations. Cluster analysis revealed four main clusters of chronic conditions: cardiovascular disease risk factors and conditions, age-related and metabolic conditions, tobacco-related and alcohol-related conditions and pain, musculoskeletal and psychological conditions. Similar clusters of chronic conditions have been reported in population-based studies. Although different methodologies prevent a direct comparison between them, it is remarkable that all studies on patterns of multimorbidity report a cluster of cardiovascular conditions, a cluster of metabolic conditions (whether or not this is associated with cardiovascular conditions) and a cluster of pain, depression and related conditions. ${ }^{23} 243132$ The clustering of some conditions may be due to common pathophysiological pathways, or to common risk factors, as previously shown. ${ }^{12}$ For example, a correlation between atrial fibrillation and stroke is to be expected, as atrial fibrillation is a condition that increases the risk of stroke. A similar example would be the correlation between diabetes and cardiovascular disease, as diabetes is considered a risk factor. The conditions in the third cluster (smoking, alcohol abuse and chronic obstructive pulmonary disease) and fourth cluster (pain, musculoskeletal and psychological conditions) can be explained following a similar logic. ${ }^{20}$ The association between the musculoskeletal and psychological conditions of patients attending primary care practices is well known and has been described often, although rarely in relation to multimorbidity. ${ }^{33} 34$

Such cluster analyses could be valuable to point out which conditions might be more likely to develop in patients whose current chronic conditions are known. However, to provide true added value to clinical practice, further analyses would be needed to evaluate how clustering varies with gender, age groups or socioeconomic status, for example.

Enrolling patients in GPs' private practices is one key to successfully describing multimorbidity as GPs usually lead and orchestrate the complex management of multimorbid patients. More than a quarter of the patients in our study reported that their treatment plan was managed solely by their GP, with an additional $30 \%$ reporting their GP plus one specialist. Furthermore, only $10.6 \%$ of the 


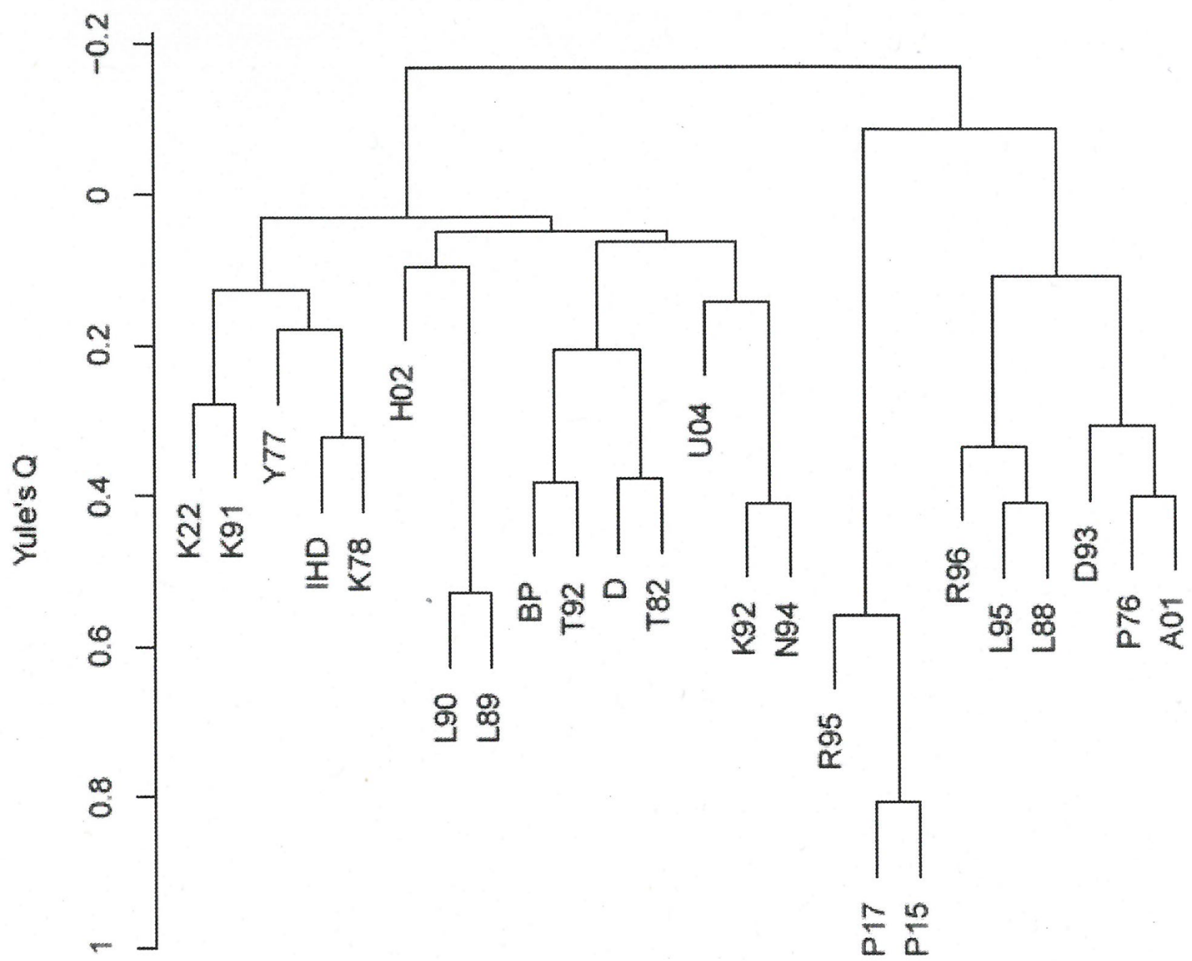

Clusters of chronic conditions

\begin{tabular}{|c|c|c|c|}
\hline 1 & $\begin{array}{l}\text { Cardiovascular risk factors and conditions } \\
\text { K22 - Risk factor cardiovascular disease } \\
\text { K91 - Cerebrovascular disease } \\
\text { Y77 - Malignant neoplasm prostate } \\
\text { IHD - Ischemic heart disease } \\
\text { K78 - Atrial fibrillation }\end{array}$ & 3 & $\begin{array}{l}\text { Alcohol and tobacco-related conditions } \\
\text { R95 - Chronic obstructive pulmonary disease } \\
\text { P17 - Tobacco abuse } \\
\text { P15 - Chronic alcohol abuse }\end{array}$ \\
\hline 2 & $\begin{array}{l}\text { Metabolic and age-related conditions } \\
\text { H02 - Hearing complaint and presbyacusis } \\
\text { L89 - Osteoarthrosis of hip } \\
\text { L90 - Osteoarthrosis of knee } \\
\text { BP - Elevated BP/hypertension } \\
\text { T92 - Gout } \\
\text { D - Diabetes } \\
\text { T82 - Obesity } \\
\text { U04 - Incontinence (urine) } \\
\text { K92 - Atherosclerosis/PVD } \\
\text { N94 - Peripheral neuritis / Neuropathy }\end{array}$ & 4 & $\begin{array}{l}\text { Pain, musculoskeletal and psychological } \\
\text { conditions } \\
\text { R96 - Asthma } \\
\text { L95 - Osteoporosis } \\
\text { L88 - Rheumatoid arthritis/seropositive arthritis } \\
\text { D93 - Irritable bowel syndrome } \\
\text { P76 - Depressive disorder } \\
\text { A01 - Pain general/multiple site }\end{array}$ \\
\hline
\end{tabular}

Figure 2 Cluster analysis, dendrogram for the conditions with a prevalence of $5 \%$ based on Yule's $Q$ values. BP, blood pressure; PVD, peripheral vascular disease.

patients reported receiving organised home care. In the Swiss healthcare system, health insurance coverage is mandatory, yet GPs do not play the role of gatekeepers and patients have the possibility of consulting a specialist directly. It is remarkable that despite this freedom of choice, GPs still play a key role in handling most multimorbid patients.

The present study has some limitations. First, the sample of GPs was a convenience sample; however, it was representative of the GPs in Switzerland. Second, using a list of 75 chronic conditions might still not represent the necessary variety and complexity of the chronic conditions seen by GPs. However, we had to restrict the list of chronic conditions-mainly so that the list would be user-friendly for GPs. The list of conditions itself has shortcomings related to the use of the ICPC-2 classification: the condition of chronic kidney disease is absent, and there is no precise item for lower-back pain, for instance. The 
experts who established the list of 75 conditions did not consider certain conditions essential (eg, thyroid disorders) in a multimorbidity context. ${ }^{16}$

We aimed to characterise a large random sample of multimorbid patients and evaluate the clustering of chronic conditions within it. Given Switzerland's ageing population and the emerging epidemic of multimorbidity in industrialised countries, accurately depicting the multiple expressions of multimorbidity in family practices' patients should be a high priority. Indeed, it is particularly important because most treatment guidelines have been written for single chronic conditions. Although some newer guidelines have tried to include the clinical implications of multiple chronic conditions, they are usually limited to the most prevalent ones and fail to account for the diversity of chronic conditions that can be seen in primary care. Although our study does not include direct clinical implications, it provides a better understanding of the diversity of possible multimorbid profiles, which can be encountered in primary care and should inform further developments in clinical guidelines for patients with multiple chronic conditions.

Acknowledgements We would like to thank the $100 \mathrm{GPs}$ who participated in this study for their valuable feedback, as well as all the participating patients.

Contributors ADL, AAN, NS, PB, BB, DW, RT, PF, AZ, DMH, TR and LH developed the protocol and defined the studyât ${ }^{\mathrm{TM}}{ }_{\mathrm{S}}$ objectives. ADL, AAN, NS, PB, BB, DW, PF, RT, $\mathrm{DMH}, \mathrm{AZ}, \mathrm{TR}$ and $\mathrm{LH}$ defined the list of variables to be collected. ADL, AAN, BB, PB, NS, DW, RT, PF and LH developed the questionnaires in French and German. LH, AZ, SE, DMH and PF help recruiting the participating GPS. ADL and JP conducted the statistical analyses. All authors contributed to the development and revision of the manuscript and the decision to submit for publication.

Funding The Swiss University Conference granted funding to reinforce teaching and research in primary care in Switzerland. Within the frame of this financial support, the five Swiss institutes for family medicine (Basel, Bern, Geneva, Zurich and Lausanne) collaborate under the denomination: Swiss Academy for Family Medicine (SAFMED). Part of the funding is to be used for national research projects in primary care.

\section{Competing interests None declared.}

Ethics approval Human Research Ethics Committee of the Canton Vaud acting as the lead ethics committee for Switzerland (Protocol 315/14).

Provenance and peer review Not commissioned; externally peer reviewed.

Data sharing statement At present, no other unpublished data are available.

Open Access This is an Open Access article distributed in accordance with the Creative Commons Attribution Non Commercial (CC BY-NC 4.0) license, which permits others to distribute, remix, adapt, build upon this work non-commercially, and license their derivative works on different terms, provided the original work is properly cited and the use is non-commercial. See: http://creativecommons.org/ licenses/by-nc/4.0/

(c) Article author(s) (or their employer(s) unless otherwise stated in the text of the article) 2017. All rights reserved. No commercial use is permitted unless otherwise expressly granted.

\section{REFERENCES}

1. Van den Akker MBF, Knottnerus JA. Comorbidity or multimorbidity: what's in a name? A review of literature. Eur J Gen Pract 1996;2:65-70.

2. Barnett K, Mercer SW, Norbury M, et al. Epidemiology of multimorbidity and implications for health care, research, and medical education: a cross-sectional study. Lancet 2012;380:37-43.

3. Bähler $\mathrm{C}$, Huber $\mathrm{CA}$, Brüngger $\mathrm{B}$, et al. Multimorbidity, health care utilization and costs in an elderly community-dwelling population: a claims data based observational study. BMC Health Serv Res 2015;15:23.

4. Pache B, Vollenweider P, Waeber G, et al. Prevalence of measured and reported multimorbidity in a representative sample of the Swiss population. BMC Public Health 2015;15:164.

5. Rizza A, Kaplan V, Senn O, et al. Age- and gender-related prevalence of multimorbidity in primary care: the Swiss FIRE project. BMC Fam Pract 2012;13:113.

6. Streit S, da Costa BR, Bauer DC, et al. Multimorbidity and quality of preventive care in Swiss university primary care cohorts. PLoS One 2014;9:e96142.

7. Schäfer I, Hansen H, Schön G, et al. The influence of age, gender and socio-economic status on multimorbidity patterns in primary care. First results from the multicare cohort study. BMC Health Serv Res 2012;12:89.

8. Fortin M, Stewart M, Poitras ME, et al. A systematic review of prevalence studies on multimorbidity: toward a more uniform methodology. Ann Fam Med 2012;10:142-51.

9. Bayliss EA, Bayliss MS, Ware JE, et al. Predicting declines in physical function in persons with multiple chronic medical conditions: what we can learn from the medical problem list. Health Qual Life Outcomes 2004;2:47.

10. Gijsen R, Hoeymans N, Schellevis FG, et al. Causes and consequences of comorbidity: a review. J Clin Epidemiol 2001;54:661-74.

11. Prados-Torres A, Calderón-Larrañaga A, Hancco-Saavedra J, et al. Multimorbidity patterns: a systematic review. J Clin Epidemiol 2014;67:254-66.

12. Starfield $B$. Threads and yarns: weaving the tapestry of comorbidity. Ann Fam Med 2006;4:101-3.

13. Guthrie B, Payne K, Alderson P, et al. Adapting clinical guidelines to take account of multimorbidity. BMJ 2012;345:e6341.

14. Fortin M, Contant E, Savard C, et al. Canadian guidelines for clinical practice: an analysis of their quality and relevance to the care of adults with comorbidity. BMC Fam Pract 2011;12:74.

15. Déruaz-Luyet A, N'Goran AA, Tandjung R, et al. Multimorbidity in primary care: protocol of a national cross-sectional study in Switzerland. BMJ Open 2015;5:e009165.

16. N'Goran AA, Blaser J, Deruaz-Luyet A, et al. From chronic conditions to relevance in Multimorbidity: a four-step study in family medicine. Fam Pract 2016;33:439-44.

17. Tran VT, Montori VM, Eton DT, et al. Development and description of measurement properties of an instrument to assess treatment burden among patients with multiple chronic conditions. BMC Med 2012;10:68.

18. Cohidon C, Cornuz J, Senn N. Primary care in Switzerland: evolution of physicians' profile and activities in twenty years (1993-2012). BMC Fam Pract 2015;16:107.

19. Selby K, Cornuz J, Senn N. Establishment of a representative practice-based research network (PBRN) for the monitoring of primary care in Switzerland. J Am Board Fam Med 2015;28:673-5.

20. Tandjung R, Senn O, Marty F, et al. Career after successful medical board examination in general practice-a cross-sectional survey. Swiss Med Wkly 2013;143:w13839.

21. Violan C, Foguet-Boreu Q, Flores-Mateo G, et al. Prevalence, determinants and patterns of multimorbidity in primary care: a systematic review of observational studies. PLoS One 2014;9:e102149.

22. Lochner KA, Cox CS. Prevalence of multiple chronic conditions among Medicare beneficiaries, United States, 2010. Prev Chronic Dis 2013;10:E61.

23. Marengoni A, Winblad B, Karp A, et al. Prevalence of chronic diseases and multimorbidity among the elderly population in Sweden. Am J Public Health 2008;98:1198-200.

24. Schäfer I, von Leitner EC, Schön G, et al. Multimorbidity patterns in the elderly: a new approach of disease clustering identifies complex interrelations between chronic conditions. PLoS One 2010;5:e15941.

25. Steinman MA, Lee SJ, John Boscardin W, et al. Patterns of multimorbidity in elderly veterans. J Am Geriatr Soc 2012;60:1872-80.

26. van den Bussche $H$, Schäfer I, Wiese B, et al. A comparative study demonstrated that prevalence figures on multimorbidity require cautious interpretation when drawn from a single database. J Clin Epidemiol 2013;66:209-17.

27. Brett T, Arnold-Reed DE, Popescu A, et al. Multimorbidity in patients attending 2 Australian primary care practices. Ann Fam Med 2013;11:535-42.

28. Britt HC, Harrison CM, Miller GC, et al. Prevalence and patterns of multimorbidity in Australia. Med J Aust 2008;189:72-7. 
29. Agborsangaya CB, Lau D, Lahtinen M, et al. Multimorbidity prevalence and patterns across socioeconomic determinants: a cross-sectional survey. BMC Public Health 2012;12:201.

30. Roberts KC, Rao DP, Bennett TL, et al. Prevalence and patterns of chronic disease multimorbidity and associated determinants in Canada. Health Promot Chronic Dis Prev Can 2015;35:87-94.

31. Formiga F, Ferrer A, Sanz H, et al. Patterns of comorbidity and multimorbidity in the oldest old: the Octabaix study. Eur J Intern Med 2013;24:40-4.
32. Islam MM, Valderas JM, Yen L, et al. Multimorbidity and comorbidity of chronic diseases among the senior Australians: prevalence and patterns. PLoS One 2014;9:e83783.

33. Haftgoli N, Favrat B, Verdon F, et al. Patients presenting with somatic complaints in general practice: depression, anxiety and somatoform disorders are frequent and associated with psychosocial stressors. BMC Fam Pract 2010:11:67.

34. Kroenke K, Wu J, Bair MJ, et al. Reciprocal relationship between pain and depression: a 12-month longitudinal analysis in primary care. $J$ Pain 2011;12:964-73. 